\title{
BMJ Open Evaluation of eight-style Tai chi on cognitive function in patients with cognitive impairment of cerebral small vessel disease: study protocol for a randomised controlled trial
}

\author{
Xiaoyong Zhong (D) , ${ }^{1}$ Xinghui Yan, ${ }^{2}$ Hui Liang, ${ }^{1}$ Rui Xia (D) , ${ }^{3}$ Bin Chen (D) , ${ }^{4}$ \\ Hong-Jia Zhao ${ }^{5}$
}

To cite: Zhong $X$, Yan $X$, Liang $\mathrm{H}$, et al. Evaluation of eight-style Tai chi on cognitive function in patients with cognitive impairment of cerebral small vessel disease: study protocol for a randomised controlled trial. BMJ Open 2021;11:e042177. doi:10.1136/ bmjopen-2020-042177

- Prepublication history for this paper is available online. To view these files, please visit the journal online (http://dx.doi. org/10.1136/bmjopen-2020042177).

$\mathrm{XZ}$ and $\mathrm{XY}$ contributed equally.

Received 27 June 2020

Revised 14 December 2020

Accepted 29 December 2020

Check for updates

(C) Author(s) (or their employer(s)) 2021. Re-use permitted under CC BY-NC. No commercial re-use. See rights and permissions. Published by BMJ.

For numbered affiliations see end of article.

Correspondence to Professor Hong-Jia Zhao; hongjiafz@163.com

\section{ABSTRACT}

Introduction Cerebral small vessel disease (CSVD) is a critical factor that causes cognitive decline and progresses to vascular dementia and acute cerebrovascular events. Tai chi has been proven to improve nerve plasticity formation and directly improve cognitive function compared with other sports therapy, which has shown its unique advantages. However, more medical evidence needs to be collected in order to verify that Tai chi exercises can improve cognitive impairment due to CSVD. The main purposes of this study are to investigate the effect of Tai chi exercise on neuropsychological outcomes of patients with cognitive impairment related to CSVD and to explore its mechanism of action with neuroimaging, including functional MRI (fMRI) and event-related potential (P300).

Methods and analysis The design of this study is a randomised controlled trial with two parallel groups in a 1:1 allocation ratio with allocation concealment and assessor blinding. A total of 106 participants will be enrolled and randomised to the 24-week Tai chi exercise intervention group and 24-week health education control group. Global cognitive function and the specific domains of cognition (memory, processing speed, executive function, attention and verbal learning and memory) will be assessed at baseline and 12 and 24 weeks after randomisation. At the same time, fMRI and P300 will be measured the structure and function of brain regions related to cognitive function at baseline and 24 weeks after randomisation. Recruitment is currently ongoing (recruitment began on 9 November 2020). The approximate completion date for recruitment is in April 2021, and we anticipate to complete the study by December 2021.

Ethics and dissemination Ethics approval was given by the Medical Ethics Committee of the Affiliated People's Hospital of Fujian University of Traditional Chinese Medicine (approval number: 2019-058-04). The findings will be disseminated through peer-reviewed publications and at scientific conferences.

Trial registration number ChiCTR2000033176; Preresults.
Strengths and limitations of this study

- This study will first evaluate the effect of eight-style Tai chi exercise on neuropsychological outcomes of patients with cognitive impairment related to cerebral small vessel disease (CSVD).

- This is the first study to explore the potential mechanisms underlying eight-style Tai chi induced cognitive improvement in this population.

- A prospective, randomised, controlled study with blinded outcome measurements will be performed.

- The efficacy of an eight-style Tai chi exercise intervention on cognitive impairment related to CSVD in improving cognitive ability remains to be determined.

\section{BACKGROUND}

Cerebral small vessel disease (CSVD) refers to a range of clinical, imaging and pathological syndromes involving the damage of white matter and deep grey matter in the brain caused by various small blood vessel pathological processes. CSVD accounts for 25\%-50\% of acute strokes in China, which is significantly higher than the rate in Western countries. ${ }^{1}$ More importantly, with the accelerated ageing of the population and increased incidence of CSVD, cognitive impairment of CSVD is one of the most common cognitive disorders. ${ }^{2}$ Approximately $30 \%-64 \%$ of patients with CSVD have different degrees of cognitive impairment. ${ }^{3}$ CSVD-induced vascular cognitive impairment (VCI) is the second leading cause of dementia after Alzheimer's disease. ${ }^{4}$ Moreover, CSVD plays an essential role in the pathogenesis of poststroke cognitive impairment. ${ }^{5}$ As a result, alleviation of cognitive impairment related to CSVD, especially at the early stage of cognitive decline due to CSVD, such as mild cognitive impairment (MCI) stage, and control of 
the primary disease progression will be very meaningful and has attracted increasing attention from scholars.

According to the latest clinical practice guideline for cognitive impairment of CSVD in China, comprehensive interventions (including lifestyle modifications, controlling vascular risk factors, treatment of primary vascular diseases and so on) and preventive strategies may have a modest effect at the individual level but lead to a major reduction in burden at the population level. ${ }^{2}$ Currently, the pharmacological treatment for CSVD and relevant cognitive impairment has remained controversial. Clinical evidence indicates that patients with CSVD are more likely to have long-term cerebral haemorrhage after thrombolytic therapy. ${ }^{6-8}$ Although thrombolysis or antiplatelet therapy is not an absolute contraindication for CSVD, the current treatment strategy for CSVD is mainly dependent on preventive and non-pharmacological therapy. ${ }^{6}$ By actively intervening risk factors of cerebrovascular diseases, such as lowering blood pressure, lowering blood sugar, lowering blood lipids and antiplatelet aggregation, one can effectively delay the process of cerebrovascular disease. ${ }^{3}$ Nevertheless, few primary or secondary preventive intervention studies have provided cognitive endpoints. In the SPS3 Trial (Secondary Prevention of Small Subcortical Strokes), dual antiplatelet therapy and intensive antihypertensive therapy had no significant therapeutic effect on cognitive end points. ${ }^{9}$ For the pharmacological treatment for cognitive impairment of CSVD, it is still mainly referenced to the treatment of dementia. ${ }^{10}$ Although the incidence of cognitive impairment in CSVD is high, and the social burden is heavy, relatively few studies have focused on the treatment of cognitive impairment in CSVD. At present, clinical trials have mainly focused on $\mathrm{VaD}$, and only a small number of studies have focused on subcortical VCI. Meanwhile, side effects such as nausea and vomiting, anorexia, syncope and insomnia, caused by drug treatment cannot be avoided. For the above reasons, due to the undesirable effects of drug intervention, combined with the side effects of long-term medication, non-pharmacological treatment methods have increasingly received attention. Non-pharmacological therapies, including exercise training, occupational therapy, cognitive training, music therapy and so on., have been shown to improve overall cognitive dysfunction. ${ }^{5}$ Among those nonpharmacological therapies, physical exercise has beneficial effects on neurogenesis and vascular health, which have been proved to be important measures related to preventing or delaying the progression of cerebrovascular disease and its cognitive dysfunction (level of evidence: IIb) ${ }^{11}{ }^{12}$ Many studies have shown that exercise therapy can enhance the brain's functions by stimulating neurons from multiple pathways, thus improving cognitive function. ${ }^{13-18}$ However, classic aerobic training mainly improves cognition by maintaining exercise intensity and improving cardiac function. ${ }^{19}$ Moreover, high-intensity interval exercise will bring more risk to the CSVD population. Traditional Chinese exercises (such as Tai chi) act as a kind of mind-body exercise therapy due to the combination of particular exercise intensity with sophisticated movement training as well as breath-control practice. Traditional Chinese exercises have been proved to improve nerve plasticity formation and directly modestly improve cognitive function compared with other sports therapy, which has shown its unique advantages. ${ }^{20} 21$

Tai chi integrates the characteristics of physical and mental medicine and embodies the traditional Chinese medicine rehabilitation theory of 'integration of form and spirit'. Because it is slow and relatively safe, it is especially suitable for older people to practice, and this exercise has become one of the traditional representative exercises in China. There is abundant clinical evidence to suggest that Tai chi exercise can improve cognitive function, improve balance, control blood pressure, prevent falls and improve quality of life. ${ }^{22-27}$ However, Tai chi exercise still lacks reliable medical evidence for improving cerebral small blood vessel-related cognitive dysfunction, and its underlying mechanism still needs to be revealed. Meanwhile, traditional Tai chi has some limits, such as it is challenging to standardise exercises and popularise. For the purpose of streamlining exercise prescription, this study intends to use eight-style Tai chi as an intervention measure. The eight styles of Tai chi draw on the eight major and basic movements of Yang style tai chi, the content is refined, the focus is prominent, it is easy to learn and remember and it is easy to promote to the community.

Currently, a promising brain functional imaging technique, resting-state functional MRI (fMRI), has been widely used in human brain functional network research; it can show metabolism in different areas, spontaneous activity in different mode regions, and intraregional or inter-regional connectivity among different brain networks. ${ }^{28}$ Previous studies have suggested that mind-body exercise improves cognitive function and modulates the function and structure of the hippocampus and anterior cingulate cortex in patients with MCI. ${ }^{21}$ Meanwhile, event-related potential (ERP) P300 amplitude is suggested to be a useful marker for the detection of preclinical changes before the appearance of cognitive and structural deterioration. ${ }^{29}$ These findings endorse fMRI and P300 as useful tools to measure cognitive function objectively.

From the above, the primary purpose of this study is to conduct a methodologically rigorous randomised controlled trial (RCT) to systematically investigate the effect of Tai chi exercise on neuropsychological outcomes of patients with CSVD and to explore its mechanism of action from neuroimaging based on fMRI and ERP (P300). We hypothesise that patients with CSVD with MCI who receive a 24-week Tai chi training intervention will achieve more significant improvement in specific domains of cognition and will show more favourable changes in the structure and function of related brain regions, than those who maintain their usual physical activities and receive primary prevention education regarding cerebrovascular disease.

\section{Study aims}

The primary aims of this study are to evaluate the modulation effect of a 24-week eight-style Tai chi training in patients with MCI due to CSVD and to explore the underlying mechanism based on fMRI and ERP. 
Meanwhile, the secondary aims of this study are as follows:

1. To investigate the effect of Tai chi on specific domains of cognition in patients with MCI due to CSVD.

2. To explore the mechanism of action of Tai chi on cognitive function in patients with MCI due to CSVD based on fMRI and cerebrovascular function.

3. To investigate the effect of Tai chi on high-risk factors (serum lipid parameter, fasting plasma glucose ( FPG ), homocysteine, blood pressure, body mass index and waist-to-hip ratio) for CSVD.

4. To investigate the effect of Tai chi on ERP (P300) in patients with MCI due to CSVD.

\section{METHOD/DESIGN}

\section{Study design}

The design of this study is an RCT with two parallel groups in a 1:1 allocation ratio with allocation concealment and assessor blinding. The design follows the Standard Protocol Items Recommendations for Interventional Trials (SPIRIT) ${ }^{30}$ and the Consolidated Standards of Reporting Trials (CONSORT) ${ }^{31}$ A total of 106 eligible participants will be enrolled from the Affiliated People's Hospital of Fujian University of Traditional Chinese Medicine or the Community Center in a different District of Fuzhou City, China. The participants will be randomised into two groups: the 24-week eight-style Tai chi exercise intervention group ( $30 \mathrm{~min}$ per session, three sessions per week) or the 24-week usual activity control group with primary prevention education regarding cerebrovascular disease and cognition impairment. Global cognitive function and specific domains of cognition (memory, processing speed, executive function, attention and verbal learning and memory) will be assessed at baseline before randomisation and at 12, 24 weeks (at the end of intervention). Furthermore, both the structure and function of brain regions related to cognitive function and haemodynamic variables will be measured using fMRI and ERP (P300), and cerebrovascular risk factors (serum lipid parameter, FPG, homocysteine, blood pressure, body mass index, waist-to-hip ratio) will be measured at baseline before randomisation and at 24 weeks. All data collectors will be blinded to the group assignments. The flow diagram for this trial is presented in figure 1.

\section{Study population}

The study population is patients with a diagnosis of MCI of CSVD living in the community in Fuzhou City. The diagnostic, inclusion and exclusion criteria for the study sample are as follows.

\section{Diagnostic criteria}

At present, according to the guidelines related to VCI, CSVD is diagnosed by aetiology or classification. ${ }^{32}$ Therefore, according to current clinical practice guidelines for cognitive impairment of CSVD in China (2019), ${ }^{2}$ the diagnosis of cognitive impairment of CSVD must be met: (i) the establishment of the presence of cognitive

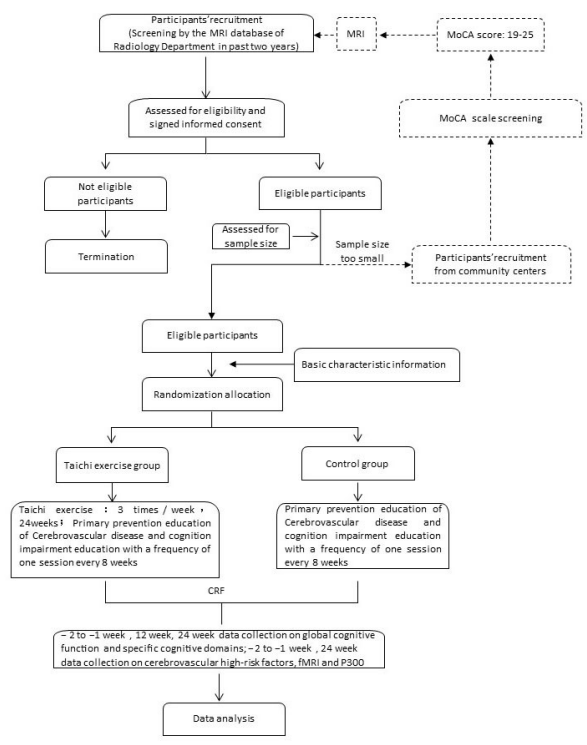

Figure 1 Flow diagram of the study design.

impairment and (ii) the determination that CSVD is the dominant pathology that accounts for the cognitive deficits. The specific diagnostic criteria for cognitive impairment due to CSVD are listed in table $1 .^{2}$

\section{Inclusion criteria}

The eligible participants must meet the following criteria: (1) match the diagnostic criteria of cognitive impairment of CSVD (according to the Clinical practice guideline for cognitive impairment of CSVD of China $(2019)) ;^{2}$ (2) Montreal Cognitive Assessment (MoCA) score: 19-25 (two points are added if the subject has $\leq 6$ years of education; one point is added if the subject has $>6$ and $\leq 12$ years of education, no points are added for the subject with $>12$ years of education) $;^{33}$ (3) aged 50 years or older; (4) no regular physical exercise for at least half a year (regular exercise means exercise with a frequency of at least three times a week and at least 20 min per session) and (5) the subject has signed an informed consent.

\section{Exclusion criteria}

Exclusion criteria include the following: (1) there are other causes of cognitive impairment, such as Alzheimer's disease, Lewy body dementia, frontotemporal dementia, Parkinson's disease, tumours, hydrocephalus, trauma, syphilis, acquired immune deficiency syndrome, Creutzfeldt-Jakob disease; (2) cortical or cortical/subcortical mixed lesions, subcortical non-lacunar infarcts, haemorrhagic lesions; (3) white matter lesions caused by other diseases such as multiple sclerosis, sarcoidosis, brain radiotherapy; (4) current severe medical conditions for which exercise is contraindicated, such as severe cardiovascular, renal, hepatic or musculoskeletal diseases; (5) previous medical records or clinicians or family members have confirmed that they have previously suffered from cognitive impairment-related diseases or have used drugs for cognitive impairment before onset; (6) dementia (Global Deterioration Scale (GDS) grades 
Table 1 Diagnostic criteria for cognitive impairment of CSVD

\begin{tabular}{ll}
\hline Items & Evidence \\
\hline $\begin{array}{l}\text { Cognitive impairment: } \\
\text { subjective cognitive }\end{array}$ & $\begin{array}{l}\mathrm{MCl} \text { (i) cognitive decline in one or more cognitive functional domains; (ii) cognitive impairment is not } \\
\text { decline; objective }\end{array}$ \\
$\begin{array}{l}\text { evidence of cognitive } \\
\text { impairment }\end{array}$
\end{tabular}

Presence of neuroimaging (MRI) evidence of CSVD (one of the following)

Identification of CSVD as evidence of cognitive impairment

(i) Multiple lacunar infarctions in white matter and deep grey matter; (ii) ischaemic white matter lesions; (iii) enlarged perivascular space; (iv) cortical microinfarction and cerebral microbleed.

Clinical evidence: (i) documented history of CSVD, with cognitive decline temporally associated with the event; (ii) evidence for decline is prominent in speed of information processing, complex attention and/ or frontal-executive functioning. One of the following features is additionally present: early gait disorder, early urination control disorder (which cannot be explained by urologic disease), personality and mood changes.

Imaging evidence of CSVD is sufficient to explain the existence of cognitive impairment: (i) multiple lacunar infarcts $(>2)$ outside the brainstem; one to two lacunes may be sufficient if strategically placed or in combination with extensive white matter lesions; (ii) extensive and confluent white matter lesionextending periventricular and deep white matter lesions: extending caps $(>10 \mathrm{~mm}$ as measured parallel to ventricle) or irregular halo ( $>10 \mathrm{~mm}$ broad, irregular margins and extending into deep white matter) and diffusely confluent hyperintensities ( $>25 \mathrm{~mm}$, irregular shape) or extensive white matter change (diffuse hyperintensity without focal lesions), and lacune(s) in the deep grey matter; (iii) enlarged perivascular space: large sample studies were required to provide corresponding evidence (there is evidence showing that the expansion of the perivascular space with a short axis of subcortical diameter $>3 \mathrm{~mm}$ increases the risk of vascular dementia; ${ }^{62}$ (iv) cortical microinfarction and cerebral microbleed: large sample studies were required to provide corresponding evidence (evidence has shown that patients with $>3$ cerebral microinfarction can have significant cognitive decline; ${ }^{63}$ the number of cerebral microbleeds $\geq 3$, regardless of location, are associated with dementia and vascular dementia. ${ }^{64}$

CSVD, cerebral small vessel disease; $\mathrm{MCl}$, mild cognitive impairment.

II-III), cannot take care of themselves (Activity of Daily Living(ADL) Scale $<20$ points), severe mental illness, epilepsy, severe depression (Baker Depression Scale $>10$ points); (7) history of alcohol intoxication, drug addiction; (8) contraindication for MRI examination and (9) participating in other clinical trials that influence the evaluation of the results of this study.

\section{Recruitment and screening}

Participants will be first recruited from the Affiliated People's Hospital of Fujian University of Traditional Chinese Medicine by the blinded research assistants (using a filter in the MRI database from the Department of Radiology over the past 2 years). We expect a possible recruitment rate at $60 \%-70 \%$. If there are not enough participants, we will recruit the rest of the participants from within different District centres of Fuzhou City by posting posters, sending leaflets and brochures and setting up a recruiting station at the corresponding community centre. Potential participants will first complete a screening questionnaire and assessment of cognitive function to determine their eligibility. Eligible participants will receive information about this trial and have an informed discussion with trained research assistants regarding the information provided. Those who are interested in participating will have the baseline assessment of outcomes arranged. Research assistants will obtain written informed consent before the baseline assessment.

\section{Randomisation, allocation concealment and blinding}

After the baseline assessment, the eligible participants will be randomly allocated with an equal rate to their respective groups. The random allocation sequence will be produced using the random number generators of the statistical software SPSS 25.0 and will be managed by an independent statistician blinded to the recruitment, evaluation and intervention of the participants. The research assistants will request (via telephone) a participant's allocation from the statistician. We cannot mask participants, exercise coaches or intervention supervisors to the allocated treatment, but outcome assessors and data statisticians will be blinded to group allocation.

\section{Patient and public involvement}

Patients and public were not involved in the design of this study.

\section{Intervention}

Tai chi exercise group: Participants in the Tai chi exercise group will receive 24 weeks of eight-style Tai chi exercise training with a frequency of 3 days a week and $30 \mathrm{~min}$ a day, including 5 min warm-up, 20 min Tai chi training 
and 5 min cool down. Participants will also receive health education about cognition impairment as well as primary prevention education regarding cerebrovascular disease once every 8 weeks ( $30 \mathrm{~min}$ per session). Tai chi training will be assigned at $2-3$ community centres with $20-35$ individuals per centre. Professional coaches, who have been engaged in teaching Tai chi exercise to college students at the Fujian University of Traditional Chinese Medicine (FJTCM), will be employed to guide participants' training. The training scheme of eight-style Tai chi exercise is planned according to the General Administration of Sport of China and consists of 10 postures (including the preparation and ending posture) ${ }^{34} \mathrm{~A}$ 'Eight-style Tai chi practice Manual' will be applied to standardise the exercise training between different coaches. A heart rate (HR) monitor will be used to monitor participants' energy consumption and HR during training. A Tai chi training record log will be applied to record HR, training time and completion degree.

Control group: Participants in the control group will not receive any specific exercise intervention other than primary prevention education regarding cerebrovascular disease and cognition, with a frequency of one session every 8 weeks ( $30 \mathrm{~min}$ per session), as in the training groups. They will maintain their original physical activity habits, while controlling vascular risk factors or treatment of primary vascular diseases. All participants will be advised not to seek any other regular exercise during the trial period. To observe any impact of the participants' usual physical activity, we will require all participants to record information about their usual daily physical activity at 1, 12 and 24 weeks of the intervention period on a daily activity $\log$.

To maximise adherence to the study protocol, the exercise intervention will be supervised, and participants exercise in groups with a possibility to interact and socialise with each other. In addition, some incentive strategy will be applied, such as health consultation for free, and provide some rewards with full adherence.

\section{Outcome assessment}

The variables in this trial consisted of basic characteristics, primary outcomes and secondary outcomes. The basic characteristics will be measured at baseline (1-2 weeks before randomised allocation). Primary and secondary outcomes will be measured at baseline, and at the end of the intervention period ( 25 weeks after randomisation). In addition, the outcomes of cognitive function, including global cognitive function and specific cognitive domains (ie, memory, processing speed, executive function, attention and verbal learning and memory), will be assessed at 12 weeks into the intervention period. The experienced staff will assess all primary and secondary outcomes at the Affiliated People's Hospital of Fujian University of Traditional Chinese Medicine and will be blinded to the allocation results of participants. A summary of all measurements in this trial is shown in table 2.

Table 2 Outline and timelines of the assessments

\begin{tabular}{|c|c|c|c|}
\hline \multirow[b]{2}{*}{ Item } & \multirow{2}{*}{$\begin{array}{l}\text { Before randomisation } \\
-2 \text { to }-1 \text { week }\end{array}$} & \multicolumn{2}{|c|}{ Intervention period (1-24 weeks after randomisation) } \\
\hline & & 12 weeks & 24 weeks \\
\hline Eligible assessment & $x$ & & \\
\hline Inclusion criteria & $x$ & & \\
\hline Exclusion criteria & $x$ & & \\
\hline \multicolumn{4}{|l|}{ Primary outcomes } \\
\hline Global cognitive function & $x$ & $x$ & $x$ \\
\hline \multicolumn{4}{|l|}{ Secondary outcomes } \\
\hline Execution & $x$ & $x$ & $x$ \\
\hline Memory & $\times$ & $x$ & $\times$ \\
\hline Visuoconstructive ability & $\times$ & $x$ & $\times$ \\
\hline $\begin{array}{l}\text { Structure and function of related brain } \\
\text { regions }\end{array}$ & $\times$ & & $\times$ \\
\hline Event-related potential (P300) & $x$ & & $\times$ \\
\hline Cerebrovascular risk factors & $\times$ & & $x$ \\
\hline
\end{tabular}

The $\times$ indicates at which point of the trial the respective assessments will take place. 


\section{Basic characteristics}

Participants' demographic characteristics (eg, age, sex, education, marital status, living arrangements, occupation and socioeconomic status) and history of disease and medication use will be collected via the electronic medical record or by the recruiters using the self-designed questionnaire. The basic MoCA score, GDS score, ADL scores and depression score will be assessed using the corresponding standardised scales. Baseline assessment will be completed before randomisation.

\section{Primary outcomes}

Global cognitive function will be measured using the MoCA scale, which is a cognitive screening instrument created and validated to detect MCI. ${ }^{35}$ MoCA is a brief (approximately $10 \mathrm{~min}$ ) test that evaluates visuospatial/ executive functions, naming, verbal memory registration, learning, attention, abstraction, $5 \mathrm{~min}$ delayed verbal memory and orientation with a total score of 0-30 (a higher score equals better function). The Chinese version of MoCA (Beijing version) has been revised by Wang Wei and Jie Hengge in $2006^{36}$ and is widely used in China with good validity, reliability and sensitivity. ${ }^{37}$ According to Tan's study, ${ }^{33}$ two points are added if the subject has $\leq 6$ years of education; one point is added if the subject has $>6$ and $\leq 12$ years of education, no points are added for the subject with $>12$ years of education.

\section{Secondary outcomes}

Executive function will be measured using the Trail Making Test (TMT). The TMT consists of two parts (TMT-A and TMT-B): TMT-A requires individuals to sequentially connect 25 encircled numbers on a sheet of paper, while TMT-B requires participants to draw a line alternating between numbers and letters, both of which must be connected in ascending order. ${ }^{38}$ TMT-A places few cognitive demands on participants and primarily captures processing speed, while TMT-B requires more complex attention shifting, planning and concentration. The Trail $\mathrm{B} / \mathrm{A}$ ratio is taken as a valid index of executive function. ${ }^{39}$

Memory will be evaluated using the Auditory Verbal Learning Test (Huashan version), which was adapted by the Guo $\mathrm{QH}^{40}{ }^{41}$ team based on the California Verbal Learning Test and Hong Kong Auditory Verbal Learning Test. This is a well-recognised measure for testing a person's ability in different stages of working memory, which include encoding, combining, storing and recovering verbal information. ${ }^{42-44}$ Specifically, we will ask the subjects to recall the words in advance. Then, the evaluator will read 12 Chinese words, including clothing, occupation and flowers. There will be four words per category. Different categories of words will be randomly, but fixedly, presented. The evaluator will read the two words at a constant interval. After reading all the words, the subject will be asked to recall the words immediately. This process will be repeated three times, and the number of words recorded by the subjects will be recorded separately. This stage will be called instant recall. After 5 min of participation in other non-language tests, the subjects will be asked to have a fourth recall of the above 12 words. At this time, the evaluator will no longer rephrase the words. After another $20 \mathrm{~min}$ of participation in other nonlanguage tests, the subject will recall the above 12 words for the fifth time. At this point, we will finish short-term delayed recall and long-term delayed recall. Then, we will use the category as a clue to give the subject a hint, as the sixth recall, that is, the clue recollection. Finally, the evaluator will read 24 words, including 12 target words and 12 disturbing words, and ask the subject to recognise whether they have learnt the word or not. This recognition test has been widely used in the clinic.

Processing speed will be evaluated by the digit symbol substitution test (DSST). ${ }^{45}$ DSST is a brief test that has nine numbers from 1 to 9 , with nine corresponding symbols. Participants are required to draw each symbol under its corresponding number within a $90 \mathrm{~s}$ time limit. The score is the number of correct answers in $90 \mathrm{~s}$ to 120 $\mathrm{s}$, and higher DSST scores mean better functioning of processing speed.

Attention will be measured by the digit cancellation test. ${ }^{46}$ The test asks subjects to cross out the number ' 6 ' in a matrix as quickly as possible within $5 \mathrm{~min}$. When the 5 min time out, the testers will record 'all crossed out', 'wrong crossed out' and 'missed crossed out' numbers and calculate the attention index. The higher the index is, the higher the level of attention.

Visuoconstructive ability will be measured by the clock drawing task (CDT). The CDT has been useful for the documentation of visuospatial processing performance (sometimes referred to as visuoconstructive ability) and is a common neuropsychological measure sensitive to cognitive changes and functional skills (eg, driving test performance) among older adults. ${ }^{47-49}$ In this task, participants are instructed to 'Draw the face of a clock showing the numbers, and the two hands, set to 10 after 11', and scoring involves 'one point each for an approximately circular face, the symmetry of number placement, and correctness of numbers'.

Structure and function of related brain regions will be measured using fMRI, which is a non-invasive method for examining brain activity and structure. T1-weighted structural images are acquired with the three-dimensional magnetisation-prepared rapid acquisition gradient-echo (3DMPRAGE) sequence (164 slices; field of view (FOV) $240 \mathrm{~mm}$; slice thickness $1 \mathrm{~mm}$; inversion time $450 \mathrm{~ms}$; echo time $30 \mathrm{~ms}$; flip angle (FA) $150^{\circ}$; matrix $256 \times 256$ ). The resting state sequence will be performed (axial (nonoblique); 42 slices; FOV $200 \mathrm{~mm}$; $3 \mathrm{~mm}$ slices with $0.6 \mathrm{~mm}$ gap; repetition time $2100 \mathrm{~ms}$; echo time $30 \mathrm{~ms}$; FA $90^{\circ}$; acquisition matrix $64 \times 64$; $3.125 \times 3.125 \mathrm{~mm}$ in plane resolution). The fMRI scan will be measured by professional operators at the Affiliated People's Hospital of FJTCM using a $3.0 \mathrm{~T}$ signal MRI scanner (SIEMENS Healthcare, Siemens, Erlangen, Germany) with a birdcage head coil. $^{50-52}$ 
P300 recordings will be performed using a two-channel device (KEYPOINT, DANTES, Wirral, England). Auditory evoked potential P300 will be used to elicit ERP. The auditory stimuli are presented in a random sequence with target tones of $2000 \mathrm{~Hz}$ occurring $20 \%$ of the time and standard tones of $1000 \mathrm{~Hz}$ occurring $80 \%$ of the time. Tones are presented binaurally through headphones at an interstimulus interval of $1.1 \mathrm{~s}$. The subject is required to distinguish between the two tones by responding to the target (moving the index finger of their right hand) and not responding to the standard. ${ }^{53}$ ERPs are digitally filtered. Peak latencies of components will be calculated relative to stimulus onset. Amplitudes of stimulus-evoked potentials are defined relative to a $100-\mathrm{msec}$ baseline period immediately before stimulus presentation. The P300 will be measured from the Pz site and identified as the largest positive peak between 280 and $450 \mathrm{~ms}^{29}$

High-risk factors (serum lipid parameter, FPG, homocysteine, blood pressure, body mass index, waist-to-hip ratio) for cerebral vessel disease will be detected at -2 to -1 week and 24 weeks after the intervention.

\section{Safety measurements}

Any unexpected adverse events during the intervention period will be monitored by supervisors, reported to a research assistant, and adverse event causality relating to the Tai chi exercise intervention and the severity of adverse events will be analysed. If muscle strain, hypoglycaemic, dizziness, heatstroke, muscle aches and so on occur during training, the supervisors will terminate the training as soon as possible, give preliminary professional treatment and provide medical advice. Serious adverse events will be reported to the ethics committee immediately. Because the intervention is a simple exercise rather than untested drugs or medical devices, supervisors instead of composition of data monitoring committee will be applied in this trail, neither auditing nor an interim analysis are planned during the trial.

\section{Sample size}

Sample size was calculated based on improvement in MoCA scores. According to previous meta-analysis results, the effect size of aerobic exercise intervention on the cognitive function of patients with MCI is approximately $0.54-0.580 .{ }^{54} \mathrm{We}$ expect at least 0.54 effect size of this outcome after the 24-week Tai chi exercise intervention. A sample size of 88 participants has been calculated to be sufficient to detect the target effect size with a type 1 error of $5 \%(\alpha=0.05)$ and $80 \%$ power $(\beta=0.20)$ by Gpower 3.1.9.2 software. Considering a $20 \%$ attrition rate, a total of 106 participants is necessary, with 53 participants in each group.

\section{Statistical analysis}

Statistical analyses will be performed using the SPSS V.25.0 software package, with a statistical significance of a twosided $\mathrm{p}<0.05$. An intention to treat (ITT) analysis, being in line with the CONSORT statement, will be used to analyse primary and secondary outcomes, and a multiple imputation method will be used to fill in missing data. Five complete datasets will be created after imputation. Each of the imputed datasets will be separately analysed, and the results of five analyses will be summarised using Rubin's rules. ${ }^{55}$ Continuous variables will be expressed as the mean (SD) for normal distribution or median (IQR) for non-normal distribution, and categorical variables will be expressed as frequencies or percentages.

Baseline characteristics among the three groups will be compared using one-way analysis of variance or the Kruskal-Wallis $\mathrm{H}$ test for continuous variables, and Pearson $\chi^{2}$ or Fisher's exact test for categorical variables. If incomparability appears, the inequality factors will be treated as confounding variables in the final efficacy analysis.

For the analysis of primary and secondary outcomes, the mixed-effects linear model with restricted maximum likelihood will be used to analyse the effect of the intervention on the continuous variables, and logistic regression models will be used for dependent categorical variables. In each model, the independent variables are the group (Tai chi group, control group), time (different measurement time points such as baseline, postintervention), confounding factors (eg, age, education) and any inequality factors among groups at the baseline assessment. The posthoc comparison between groups at different time points will be conducted using multiple comparisons with adjustment to the type 1 error rate.

The resting fMRI data will be analysed by independent component analysis and voxel-based morphometry to detect the strength of the correction coefficients for the brain regions related to cognitive function, which include the praecuneus/posterior cingulate cortex, medial prefrontal cortex, medial temporal lobe, angular gyrus, lateral temporal cortex and medial, lateral and inferior parietal cortex and hippocampus. The multiple comparisons among the two groups will be performed using the random-effects general linear model analysis. The correlation of blood oxygen level dependent signal changes between groups will be analysed using Pearson or Spearman linear correlations. Adverse events will be listed and analysed using a $\chi^{2}$ or Fisher's exact test, and severe adverse events will be listed in detail.

\section{Data collection and management}

The data will be collected by the outcome assessors using print-based case report forms (p-CRFs). Then, the p-CRFs will be transcribed into web-based case report forms (w-CRFs) with an electronic data capture system (EDC) by research assistants. A data management system based on the EDC system and web servers will be provided by the Affiliated People's Hospital of Fujian University of Traditional Chinese Medicine using a medical research platform (http://218.85.55.50:9001/) and will meet the available standards of security. Participants' data in w-CRFs will be stored in the EDC system at separate password-protected locations. The research assistants 
will be responsible for ensuring the integrity and accuracy of all data when data are transcribed into w-CRFs by means of checks on value ranges and logical checks. The development and validation of the study database, quality control and extraction of data will be performed according to the procedures of the medical research platform. The responsibilities $\log$ will identify all trial personnel responsible for data collection, data entry and handling and management of the database.

\section{Ethics issues}

This protocol will be conducted in accordance with the Declaration of Helsinki. Ethics approval has been obtained from the Medical Ethics Committee of the Affiliated People's Hospital of Fujian University of Traditional Chinese Medicine (approval number: 2019-058-04). The study protocol has been registered and is available on the Chinese Trial Registry website (registered in ChiCTR. org with the identifier ChiCTR2000033176). All participants will provide voluntary written, informed consent after a full discussion about the potential benefits and risks before participation. This study will be published in scientific journals to target a wide range of groups and presented at national conferences in the field of cognitive rehabilitation. Study results will also be sent to study participants and disseminated to researchers, healthcare providers, healthcare professionals and the general public through courses, presentations and the internet regardless of the magnitude or direction of effect.

\section{DISCUSSION}

Cerebrovascular disease is an essential factor leading to cognitive decline and progression to vascular dementia and acute cerebrovascular events. However, due to unclear clinical symptoms and various pathological changes, there are currently no unified clinical guidelines for the treatment or delay of the cognitive decline caused by CSVD. Primary prevention is still the best way to slow down its progress. Much medical evidence shows that exercise therapy is an important measure to delay the progress of cerebrovascular disease and the resulting cognitive dysfunction. Classic aerobic training usually improves cognitive function indirectly by maintaining exercise intensity and improving heart function. Nevertheless, traditional Chinese exercises (such as Tai chi), as a kind of exercise therapy due to the combination of a particular exercise intensity with sophisticated movement training, can improve nerve plasticity and directly improve cognitive function compared with other sports therapy, such as aerobic exercise, which has shown its unique advantages. ${ }^{20} 21$

An increasing number of studies have shown that an exercise (especially aerobic exercise) intervention may be beneficial for maintaining or even improving cognitive function in older adults with or without cognitive impairment. However, the optimal workload related to the type, frequency, intensity and duration of the exercise sessions has not been clearly established. ${ }^{56}$ Tai chi exercise is an aerobic exercise characterised by simple, slow, relaxing movements. Tai chi is a type of traditional Chinese Qigong exercise characterised by gentle physical movement and meditation as well as breathing. ${ }^{578}$ As a no-contact, moderate-impact, mind-body exercise, Tai chi has been practised for several hundred years in China to promote health in the general population and, particularly, in older community-dwelling adults. A previous meta-analysis and systematic review indicated that Tai chi had a positive effect on the majority of cardiac function outcomes (eg, reduced resting heart rate, increased stroke volume and increased cardiac output), lung capacity (eg, improved forced vital capacity) and cardiorespiratory endurance and was beneficially associated with the primary prevention of stroke among middle-aged and elderly adults. ${ }^{596}$ Moreover, there is evidence that suggests that Tai chi exercise can improve cognitive function. ${ }^{21-23}$ However, the effects of Tai chi in patients with MCI of CSVD have not been revealed yet. Traditional Tai chi training consists of 24 sections of movement, and it is too complicated for patients with MCI to remember and practice. As a result, in this study, we will use simplified Tai chi training styles, which only consist of eight sections of movement, as an intervention factor to observe the effects of Tai chi in patients with MCI of CSVD.

This protocol describes a randomised two-parallel controlled trial assessing the effect of regular Tai chi exercise on cognitive function in patients with MCI due to CSVD. This trial will employ rigorous methods to reduce bias, such as randomisation, blinding of the outcome assessors and statistical analysers, sample recruiting from a community-dwelling elderly population and statistical analysis according to the ITT principle. We will also invite two qualified physical exercise teachers to serve as the Tai chi exercise coaches to ensure standardised exercise training for the participants. A specific type of aerobic exercise (ie, Tai chi exercise) with an accessible exercise scheme (30 min per day on 3 days each week for 24 weeks) will be applied as the intervention, making it possible to clearly clarify the association with cognitive function or the specific domains of cognition, such as memory, execution and attention. Furthermore, the extensive outcome assessment on several levels, involving neuropsychological testing, fMRI and P300 assessment, will make it possible to explore the mechanism of action of the Tai chi intervention on cognitive functional networks and brain electrophysiology function. This will allow the researchers to investigate the relationship between subjective scales and objective indicators. Another highlight of this trial will investigate the effect of Tai chi on high-risk factors (serum lipid parameter, FPG, Homocysteine, blood pressure, body mass index and waist-to-hip ratio) of CSVD. In addition, the proposed study will be the first to investigate the effects of Tai chi exercise on patients with MCI of CSVD. The results should help improve the cognitive function of the participants with MCI and should be generalisable to the community-dwelling older population.

A potential limitation of this protocol is that participants and exercise coaches cannot be blinded because it 
is difficult to conduct blinding in non-pharmacological trials. ${ }^{61}$ Therefore, performance bias may be inevitable, but the exercise coaches will not be involved in the recruitment, assessment and data analysis of this study. In addition, this protocol does not suggest a specific follow-up period, so the long-term effects of the eightstyle Tai chi exercise will not be observed in this trial. However, considering the 24-week intervention period, there should be enough time to observe the effects of Tai chi exercise on patients with MCI due to CSVD.

In summary, this study will be the first RCT to evaluate the impact of Tai chi exercise on patients with MCI due to CSVD. The results of this trial will help to establish the optimal approach for treating cognitive decline in patients with MCI due to CSVD and to provide reliable evidence for its application in rehabilitation with Traditional Chinese Medicine.

\section{Author affiliations}

'Department of Neurology, The Affiliated People's Hospital of Fujian University of Traditional Chinese Medicine, Fuzhou, China

${ }^{2}$ Department of Physical Education, Fujian University of Traditional Chinese Medicine, Fuzhou, China

${ }^{3}$ College of Rehabilitation Medicine, Fujian University of Traditional Chinese Medicine, Fuzhou, China

${ }^{4}$ Department of Rehabilitation, The Affiliated People's Hospital of Fujian University of Traditional Chinese Medicine, Fuzhou, China

${ }^{5}$ The Affiliated People's Hospital of Fujian University of Traditional Chinese Medicine, Fuzhou, China

Acknowledgements We thank GuoHua Zheng from the School of Nursing and Health Management, Shanghai University of Medicine \& Health Sciences, for the useful suggestions. We would like to thank AJE (www.aje.cn) for English language editing.

Contributors H-JZ, XZ and XY conceived the study, designed the study protocol and drafted the manuscript. XZ wrote the manuscript and revised and finalised the study protocol. HL and XZ participated in the coordination and implementation of the study. $\mathrm{HZ}$ and $\mathrm{XZ}$ are in charge of coordination and direct implementation. RX and $\mathrm{BC}$ helped to develop the study measures and analyses. All authors contributed to drafting the manuscript and have read and approved the final manuscript.

Funding This study is supported by the National Traditional Chinese Medicine (TCM) Clinical Research Base Fund of Fujian (Grant no. JDZX201935).

\section{Competing interests None declared.}

Patient and public involvement Patients and/or the public were not involved in the design, or conduct, or reporting, or dissemination plans of this research.

\section{Patient consent for publication Not required.}

Provenance and peer review Not commissioned; externally peer reviewed.

Open access This is an open access article distributed in accordance with the Creative Commons Attribution Non Commercial (CC BY-NC 4.0) license, which permits others to distribute, remix, adapt, build upon this work non-commercially, and license their derivative works on different terms, provided the original work is properly cited, appropriate credit is given, any changes made indicated, and the use is non-commercial. See: http://creativecommons.org/licenses/by-nc/4.0/.

\section{ORCID iDs}

Xiaoyong Zhong http://orcid.org/0000-0002-3886-5400

Rui Xia http://orcid.org/0000-0002-5941-8263

Bin Chen http://orcid.org/0000-0002-2459-1605

\section{REFERENCES}

1 Tsai C-F, Thomas B, Sudlow CLM. Epidemiology of stroke and its subtypes in Chinese vs white populations: a systematic review. Neurology 2013;81:264-72.

2 Peng D, Geriatric Neurology Group, Chinese Society of Geriatrics, Clinical Practice Guideline for Cognitive Impairment of Cerebral Small Vessel Disease Writing Group. Clinical practice guideline for cognitive impairment of cerebral small vessel disease. Aging Med 2019;2:64-73.

3 Corriveau RA, Bosetti F, Emr M, et al. The science of vascular contributions to cognitive impairment and dementia (VCID): a framework for advancing research priorities in the cerebrovascular biology of cognitive decline. Cell Mol Neurobiol 2016;36:281-8.

4 Gottesman RF, Hillis AE. Vascular cognitive impairment. Behav Neurol 2010;22:1-2.

5 Teng Z, Dong Y, Zhang D, et al. Cerebral small vessel disease and post-stroke cognitive impairment. Int J Neurosci 2017;127:824-30.

6 Griebe M, Fischer E, Kablau M, et al. Thrombolysis in patients with lacunar stroke is safe: an observational study. $J$ Neurol 2014;261:405-11.

7 Willer L, Havsteen I, Ovesen C, et al. Computed tomography-verified leukoaraiosis is a risk factor for post-thrombolytic hemorrhage. $J$ Stroke Cerebrovasc Dis 2015;24:1126-30.

8 Yan S, Jin X, Zhang X, et al. Extensive cerebral microbleeds predict parenchymal haemorrhage and poor outcome after intravenous thrombolysis. J Neurol Neurosurg Psychiatry 2015;86:1267-72.

9 Huang $\mathrm{Y}$, Cheng $\mathrm{Y}, \mathrm{Wu} \mathrm{J}$, et al. Cilostazol as an alternative to aspirin after ischaemic stroke: a randomised, double-blind, pilot study. Lancet Neurol 2008;7:494-9.

10 Zhou X, Cui G, Tseng HHL, et al. Vascular contributions to cognitive impairment and treatments with traditional Chinese medicine. Evid Based Complement Alternat Med 2016;2016:1-12.

11 Dichgans M, Leys D. Vascular cognitive impairment. Circ Res 2017;120:573-91.

12 Winstein CJ, Stein J, Arena R, et al. Guidelines for adult stroke rehabilitation and recovery: a guideline for healthcare professionals from the American heart Association/American stroke association. Stroke 2016;47:e98-169.

13 Siette J, Westbrook RF, Cotman C, et al. Age-specific effects of voluntary exercise on memory and the older brain. Biol Psychiatry 2013;73:435-42.

14 Curlik DM, Shors TJ. Training your brain: do mental and physical (MAP) training enhance cognition through the process of neurogenesis in the hippocampus? Neuropharmacology 2013;64:506-14.

15 Quaney BM, Boyd LA, McDowd JM, et al. Aerobic exercise improves cognition and motor function poststroke. Neurorehabil Neural Repair 2009;23:879-85.

16 Scherder EJA, Van Paasschen J, Deijen J-B, et al. Physical activity and executive functions in the elderly with mild cognitive impairment. Aging Ment Health 2005;9:272-80.

17 Liu-Ambrose T, Eng JJ. Exercise training and recreational activities to promote executive functions in chronic stroke: a proof-of-concept study. J Stroke Cerebrovasc Dis 2015;24:130-7.

18 Murdoch K, Buckley JD, McDonnell MN. The effect of aerobic exercise on neuroplasticity within the motor cortex following stroke. PLoS One 2016;11:e152377.

19 Andrews SC, Curtin D, Hawi Z. Intensity matters: high-intensity interval exercise enhances motor cortex plasticity more than moderate exercise. Cereb Cortex 2019.

20 Lu J, Fu W, Liu Y. Physical activity and cognitive function among older adults in China: a systematic review. J Sport Health Sci 2016;5:287-96

21 Tao J, Liu J, Chen X, et al. Mind-body exercise improves cognitive function and modulates the function and structure of the hippocampus and anterior cingulate cortex in patients with mild cognitive impairment. Neuroimage Clin 2019;23:101834.

22 Tao J, Liu J, Liu W, et al. Tai Chi Chuan and Baduanjin increase grey matter volume in older adults: a brain imaging study. J Alzheimers Dis 2017;60:389-400.

23 Tao J, Chen X, Egorova N, et al. Tai Chi Chuan and Baduanjin practice modulates functional connectivity of the cognitive control network in older adults. Sci Rep 2017;7:41581.

24 Tao J, Chen X, Liu J, et al. Tai Chi Chuan and Baduanjin Mind-body training changes resting-state low-frequency fluctuations in the frontal lobe of older adults: a resting-state fMRI study. Front Hum Neurosci 2017; 11:514.

25 Liu J, Tao J, Liu W, et al. Different modulation effects of Tai Chi Chuan and Baduanjin on resting-state functional connectivity of the default mode network in older adults. Soc Cogn Affect Neurosci 2019;14:217-24. 
26 Xia R, Qiu P, Lin H, et al. The effect of traditional Chinese Mindbody exercise (Baduanjin) and Brisk walking on the dorsal attention network in older adults with mild cognitive impairment. Front Psychol 2019;10:10.

27 Zheng G-H, Zheng X, Li J-Z, et al. Effect of tai chi on cardiac and static pulmonary function in older community-dwelling adults at risk of ischemic stroke: a randomized controlled trial. Chin J Integr Med 2019;25:582-9.

28 Sporns O, Betzel RF. Modular brain networks. Annu Rev Psychol 2016:67:613-40.

29 Hünerli D, Emek-Savaș DD, Çavușoğlu B, et al. Mild cognitive impairment in Parkinson's disease is associated with decreased p300 amplitude and reduced putamen volume. Clin Neurophysiol 2019;130:1208-17.

30 Chan A, Tetzlaff JM, Altman DG. SPIRIT 2013 statement: defining standard protocol items for clinical trials, 2013: 200-7.

31 Schulz KF, Altman DG. Statement: updated guidelines for reporting parallel grouprandomised trials 2010;2010:c332.

32 Sachdev P, Kalaria R, O'Brien J, et al. Diagnostic criteria for vascular cognitive disorders: a VASCOG statement. Alzheimer Dis Assoc Disord 2014;28:206-18.

33 Tan J-ping, Li N, Gao J, et al. Optimal cutoff scores for dementia and mild cognitive impairment of the Montreal cognitive assessment among elderly and oldest-old Chinese population. J Alzheimers Dis 2015;43:1403-12.

$34 \mathrm{Li} \mathrm{Z}$. Application of priority practice of eight-style Taijiquan in community fitness. Martial Arts Res 2019;4:53-4.

35 Nasreddine ZS, Phillips NA, Bedirian V. The Montreal cognitive assessment, MoCA: a brief screening tool for mildcognitive impairment, 2005: 695-9.

36 Huang Y-Y, Qian S-X, Guan Q-B, et al. Comparative study of two Chinese versions of Montreal cognitive assessment for screening of mild cognitive impairment. Appl Neuropsychol Adult 2019:1-6.

$37 \mathrm{Yu}$ J, Li J, Huang X. The Beijing version of the Montreal cognitive assessment as a brief screening tool for mild cognitive impairment: a community-based study. BMC Psychiatry 2012;12:156.

38 Fowler PC, Zillmer E, Newman AC. A multifactor model of the Halstead-Reitan neuropsychological test battery and its relationship to cognitive status and psychiatric diagnosis. J Clin Psychol 1988;44:898-906.

39 Arbuthnott K, Frank J. Trail making test, part B as a measure of executive control: validation using a set-switching paradigm. J Clin Exp Neuropsychol 2000;22:518-28.

40 Zhao Q, Guo Q, Liang X, et al. Auditory verbal learning test is superior to Rey-Osterrieth complex figure memory for predicting mild cognitive impairment to Alzheimer's disease. Curr Alzheimer Res 2015; 12:520-6.

41 Zhao Q, Lv Y, Zhou Y, et al. Short-term delayed recall of auditory verbal learning test is equivalent to long-term delayed recall for identifying amnestic mild cognitive impairment. PLoS One 2012; 7:e51157.

42 Hao L, Xing Y, Li X, et al. Risk Factors and Neuropsychological Assessments of Subjective Cognitive Decline (p/us) in Chinese Memory Clinic. Front Neurosci 2019;13:846.

43 Zhang S, Luo Y, Dong Z, et al. Impact of periventricular hyperintensities and cystatin $C$ on different cognitive domains in the population of non-demented elderly Chinese. J Clin Neurosci 2019;68:201-10.

44 Li P, Ding D, Ma X-Y, et al. Altered intrinsic brain activity and memory performance improvement in patients with end-stage renal disease during a single dialysis session. Brain Imaging Behav 2018;12:1640-9.
45 Jaeger J. Digit symbol substitution test: the case for sensitivity over specificity in neuropsychological testing. J Clin Psychopharmacol 2018;38:513-9.

46 Della Sala S, Laiacona M, Spinnler H, et al. A cancellation test: its reliability in assessing attentional deficits in Alzheimer's disease. Psychol Med 1992;22:885-901.

47 Hubbard EJ, Santini V, Blankevoort CG, et al. Clock drawing performance in cognitively normal elderly. Arch Clin Neuropsychol 2008;23:295-327.

48 Smedslund G, Siqveland J, Leiknes KA. Psychometric assessment of the clock drawing test. Oslo, Norway: Knowledge Centre for the Health Services at The Norwegian Institute of Public Health (NIPH), 2015.

49 Parks RW, Thiyagesh SN, Farrow TFD, et al. Performance on the clock drawing task correlates with $\mathrm{fMRI}$ response to a visuospatial task in Alzheimer's disease. Int J Neurosci 2010;120:335-43.

50 Lang S, Duncan N, Northoff G. Resting-state functional magnetic resonance imaging: review of neurosurgical applications. Neurosurgery 2014;74:453-64.

51 Catalino MP, Yao S, Green DL, et al. Mapping cognitive and emotional networks in neurosurgical patients using restingstate functional magnetic resonance imaging. Neurosurg Focus 2020;48:E9.

52 Duchesne S, Chouinard I, Potvin O, et al. The Canadian dementia imaging protocol: Harmonizing national cohorts. J Magn Reson Imaging 2019;49:456-65.

53 Didoné DD, Garcia MV, Oppitz SJ, et al. Auditory evoked potential p300 in adults: reference values. Einstein 2016;14:208-12.

54 Song D, Yu DSF, Li PWC, et al. The effectiveness of physical exercise on cognitive and psychological outcomes in individuals with mild cognitive impairment: a systematic review and meta-analysis. Int $J$ Nurs Stud 2018;79:155-64.

55 Schafer JL. Multiple imputation: a primer. Stat Methods Med Res 1999:8:3-15.

56 Paillard T. Preventive effects of regular physical exercise against cognitive decline and the risk of dementia with age advancement. Sports Med Open 2015;1:20.

57 Yeh GY. Commentary on the Cochrane review of Tai Chi for rheumatoid arthritis. Explore 2008;4:275-7.

58 Yan J-H, Gu W-J, Sun J, et al. Efficacy of Tai Chi on pain, stiffness and function in patients with osteoarthritis: a meta-analysis. PLoS One 2013:8:e61672.

59 Zheng G, Li S, Huang M, et al. The effect of tai chi training on cardiorespiratory fitness in healthy adults: a systematic review and meta-analysis. PLoS One 2015;10:e117360.

60 Zheng G, Huang M, Liu F, et al. Tai chi chuan for the primary prevention of stroke in middle-aged and elderly adults: a systematic review. Evid Based Complement Alternat Med 2015;2015:1-18.

61 Boutron I, Moher D, Altman DG, et al. Extending the CONSORT statement to randomized trials of nonpharmacologic treatment: explanation and elaboration. Ann Intern Med 2008;148:295-309.

62 Ding J, Sigurðsson S, Jónsson PV, et al. Large perivascular spaces visible on magnetic resonance imaging, cerebral small vessel disease progression, and risk of dementia: the age, Gene/Environment Susceptibility-Reykjavik study. JAMA Neurol 2017;74:1105-12.

63 van Veluw SJ, Zwanenburg JJM, Engelen-Lee J, et al. In vivo detection of cerebral cortical microinfarcts with high-resolution 7T MRI. J Cereb Blood Flow Metab 2013;33:322-9.

64 Ding J, Sigurðsson S, Jónsson PV, et al. Space and location of cerebral microbleeds, cognitive decline, and dementia in the community. Neurology 2017;88:2089-97. 